\title{
The Effects of Encumbrance and Mobility on Touch-Based Gesture Interactions for Mobile Phones
}

\author{
Alexander Ng, John Williamson and Stephen Brewster \\ School of Computing Science \\ University of Glasgow \\ alexander.ng@glasgow.ac.uk, johnh.williamson@glasgow.ac.uk, stephen.brewster@glasgow.ac.uk
}

\begin{abstract}
In this paper, we investigate the effects of mobility and encumbrance (holding objects such as shopping bags) on standard gestures commonly performed on touchscreens: tapping, dragging, spreading \& pinching and rotating clockwise \& anticlockwise when completed using a twohanded input posture. These one- and two- finger on-screen gesture inputs have become common but previous research has only examined tapping performance in everyday walking and encumbered situations. Therefore, a series of Fitts' Law style targeting tasks was designed to measure the performance of each gesture with users walking only and walking while carrying bags. The results showed that encumbrance and walking had a negative impact on each gesture in terms of accuracy except for rotational actions, which were performed well. Tapping and dragging both performed poorly which shows the input difficulties of single finger interactions when encumbered and on the move. Our findings will help designers choose the appropriate input techniques for future mobile user interfaces and apps in physically demanding contexts.
\end{abstract}

\section{Author Keywords}

Encumbrance; Fitts' Law; Mobile interactions; Touchbased gestures; Walking

\section{ACM Classification Keywords}

H.5.2. User Interfaces: Input devices and strategies

\section{INTRODUCTION}

Touchscreen interfaces provide a rich variety of on-screen gesture interactions from one-finger tapping (to select icons and buttons, for example) and dragging (to pan across a photo) to two-finger pinching \& spreading (zooming in and out of an image) and rotating (changing the orientation of a map). The growth of mobile applications (apps) used in all aspects of life and the necessity to use on-screen gestures for input mean that users interact in many different ways,

Paste the appropriate copyright/license statement here often on the move and even when physically encumbered (carrying everyday objects such as bags, boxes and personal gear $[13,19,21])$ as shown in Figure 1. Previous encumbered and walking studies $[21,22,23]$ have extensively examined basic tapping performance on mobile phones and reported a significant drop in targeting performance. However, more biomechanically complex actions such as dragging, spreading, pinching and rotating gestures are less well studied on small touchscreen mobile devices such as smartphones and no research has investigated the effects of mobility and encumbrance on their performance. Therefore, a study was designed to measure and compare the individual performance of four main touchscreen gestures: tapping, dragging, spreading \& pinching and rotating (clockwise \& anticlockwise), on a mobile phone while walking and carrying typical shopping bags.

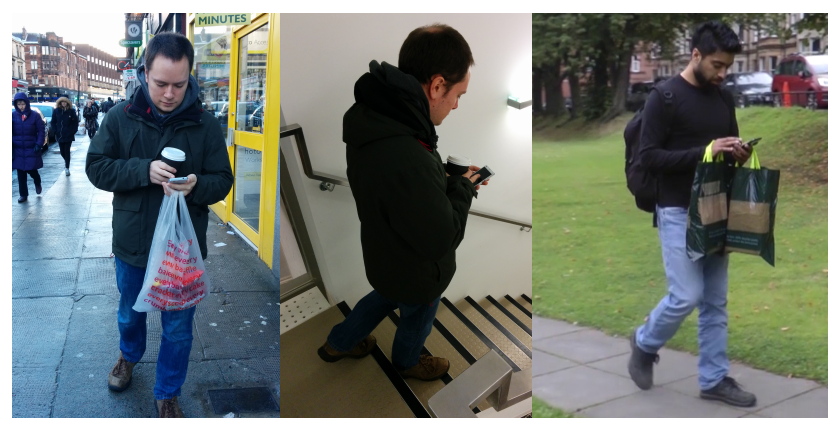

Figure 1. Typical encumbrance scenarios during mobile usage.

There are two key objectives for the study presented in this paper. Firstly, we fill a gap in the literature by extending the knowledge on encumbered tapping behaviour and assessed how well users performed both one- and two- finger on-screen gestures when situationally impaired [28]. These situations make interaction with mobile devices problematic since visual attention and mental resources are constantly interrupted by environment distractions. Furthermore, previous studies (e.g. $[6,9,10,29]$ ) that have examined onscreen gesture interactions on touchscreen devices only evaluated their effectiveness when users were in a static position and unencumbered. Therefore, it is unclear how well users can perform more complicated touch-based actions in physically challenging and awkward contexts, even though these situations are common $[13,19,21]$. Consequently, it is difficult to choose appropriate interaction techniques when users are encumbered and on the move. The results from our study can help designers make better 
decisions to improve usability with mobile devices in these contexts.

Secondly, we describe a set of abstract experimental tasks based on Fitts' Law to test each of the four different gesture inputs. Fitts' Law has been used to examine touch-based tapping [4], dragging [4] and spreading \& pinching [29]. However, there are no clear methods described in the literature that can be used to precisely quantify a user's ability to perform two-finger rotational gestures on small handheld devices. The current evaluation methods used to examine multi-digit rotational movements are for much larger computing devices such as tablets and tabletops [9,31]. These methods are less appropriate to use on smaller sized mobile devices due to limited screen space and the user's restricted input posture when walking and encumbered. Thus, we designed a new approach to measure two-finger rotational actions that is more suitable for smaller touchscreens. The set of tasks used to test all of the gesture types will give a baseline of performance and a standard method others can use in different settings or for testing newly developed interaction techniques in realistic mobile contexts.

\section{BACKGROUND}

\section{The Effects of Encumbrance and Mobility}

So far, previous encumbered studies have primarily examined the effects of encumbrance and walking on tapping performance on touchscreen mobile devices. The target acquisition study by $\mathrm{Ng}$ et al. [21] reported a significant decline in input performance when everyday objects such as bags and boxes were held while on the move. Target accuracy for selecting $4 \times 6 \mathrm{~mm}$ targets dropped from $46 \%$ to $30 \%$ when comparing unencumbered walking to holding a $3 \mathrm{~kg}$ bag in the dominant hand, for example. Later, $\mathrm{Ng}$ et al. [23] examined encumbered tapping behaviour on a touchscreen mobile phone when walking speed and walking method (ground vs. treadmill) were varied. The results from their target selection study showed that reducing walking speed by $20 \%$ only improved accuracy by $3.6 \%$ when selecting $5 x 8 \mathrm{~mm}$ targets. Accuracy was affected the most when a box was held under the dominant arm, compared to other types of encumbrances evaluated.

When input becomes too physically challenging, users are likely to change input posture to interact in a more effective manner. $\mathrm{Ng}$ et al. [22] examined tapping performance in three common postures (two-handed index finger, onehanded preferred thumb and two-handed both thumbs) while users were walking and both hands were encumbered with shopping bags. Their results showed that encumbrance had a greater effect on target accuracy and selection time than the type of input posture used since all three positions performed alike. Surprisingly, targeting performance using both thumbs was not substantially better than using the preferred thumb or index finger, despite the advantage of having an extra digit for input, which illustrates the adverse impact of encumbrance and mobility.
Oulasvirta and Bergstrom-Lehtovirta [25] examined the effects of encumbrance on different mobile devices but users in their study were not walking. A range of realworld multitasking scenarios was evaluated which included holding smaller objects than those tested by $\mathrm{Ng}$ et al. $[21,22,23]$, such as cups, pens and scissors. These objects required more intricate finger adjustments and hand grips during input. Their text entry task on a mobile phone, which compared different input modalities, showed that typing on a touchscreen was more resilient to the effects of encumbrance than using a physical keyboard or a stylus to input. However, these results are likely to differ if the user is also walking while physically constrained.

Mobile devices are often used in walking contexts and researchers have acknowledged the potential usability issues. Therefore, numerous studies have examined the impact mobility has on interactions with handheld devices. Schildbach and Rukzio [27] showed that walking caused target selection time to increase by $31 \%$ while accuracy reduced by $7 \%$ for selecting $6.74 \mathrm{~mm}^{2}$ targets with the thumb on a touchscreen mobile phone when compared to standing. In addition, interaction caused users to drop their walking speed by $27 \%$, which illustrates the cost of using mobile devices on the move. Lin et al. [15] compared stylus-based targeting on a PDA when users were standing and walking. Results from their study found that error rate was significantly higher when walking around an obstacle path than when seated but unlike [27], no significant differences were observed in terms of target selection time. Mizobuchi et al. [20] also reported that walking did not significantly affect stylus-based typing speed but error rate increased when compared to standing. Schedlbauer and Heines [26] conducted a Fitts' Law study to compare stylus-based targeting on a handheld PC between standing and walking, and found no significant differences in movement time. However, error rate was significantly higher by $11.5 \%$ when users were walking compared to when standing.

The treadmill walking study by Bergstrom-Lehtovirta et al. [3] showed that only walking between $20-40 \%$ of one's preferred walking speed (PWS) reduced target accuracy, in this case by $11 \%$. Tapping performance using the index finger began to level off when users walked between $40-$ $80 \%$ of their PWS. In addition, Bergstrom-Lehtovirta et al. found that walking speed was reduced by $24 \%$ during target selections when compared to walking alone, which was similar to the findings of [27]. Barnard et al. [2] reported a greater decrease in walking speed, between $30-37 \%$, during different reading tasks on a PDA while walking.

\section{Gestural Interactions on Touchscreens}

A number of studies have examined the performance of dragging, spreading, pinching and rotating gestures on touchscreen devices in unencumbered and non-mobile contexts. Tran et al. [29] conducted an extensive study examining spreading and pinching gestures on both a mobile phone and a tablet where users were seated. Their results 
showed that on average, one second was needed to perform the gestures on the mobile phone while pinching gestures took longer to perform than spreading actions. Findlater et al. [6] also reported that pinching was performed significantly slower than spreading, but on a tablet across different age groups. In general, older users required more time to execute pinching and spreading gestures than younger adults. Hoggan et al. [10] also investigated the performance of spreading and pinching gestures on a touchscreen tablet mimicking a multi-touch tabletop computer. In contrast to $[6,29]$, they found that pinching was faster and ergonomically easier to execute than spreading actions. Likewise, Kobayashi et al. [14] reported that pinching gestures were performed quicker than spreading for older adult users. The varied results reported by the mentioned studies between spreading and pinching when users were stationary and unencumbered makes it even more difficult to predict the performance of these gestures in walking and encumbered situations.

There has been little study of rotating gestures on mobile devices. The most related research is by Hoggan et al. [9], who examined two-finger rotational motions on a touchscreen tablet placed flat to replicate a tabletop computer. An overall mean execution time of $2.7 \mathrm{~s}$ for $90^{\circ}$ rotations was reported. Rotating clockwise took significantly longer to perform than rotating anticlockwise, although, it is worth noting that all users were right-handed. Their study also focused on the ergonomics of rotating gestures. For example, at a starting position of $0^{\circ}$ (relative to the horizontal $\mathrm{x}$-axis), rotating gestures were significantly faster than starting at $60^{\circ}$ and $120^{\circ}$. Furthermore, execution time and failure rate both significantly increased as the gap between the fingers increased. Despite these in-depth findings, it is difficult to translate their design guidelines from stationary on-table interactions to handheld devices in mobile and encumbered contexts, where the user's input posture is uncertain. We therefore examined two-finger rotational gestures in our study to fill this gap in the literature.

Findlater et al. [6] found that older adults took around 1.5s to perform dragging gestures on a tablet while younger users required less than one second. Kobayashi et al. [14] reported a higher average time of $2.17 \mathrm{~s}$ to complete dragging movements for older users and that dragging was subjectively easier to perform than tapping. Cockburn et al. [4] compared different types of input modalities for tapping and dragging on a touchscreen tablet PC. Their results showed that touch-based dragging was slower to perform than tapping but error rate was lower for dragging than tapping. However, these findings from static and unencumbered situations are likely to differ when users are walking and busy holding objects.

In summary, there is a lack of research examining the performance of gestural interactions on smaller sized touchscreen handheld devices, especially for rotating gestures. It is unclear how successfully users can execute these complex finger movements in more physically taxing contexts, such as walking and carrying bags. Therefore, we designed an experiment to test each gesture type and compared their performance in mobile and encumbered settings.

\section{EXPERIMENT}

A set of Fitts' Law style studies was designed to investigate the performance of tapping, dragging, spreading \& pinching and rotating clockwise \& anticlockwise on a touchscreen mobile phone while users were 1) walking only and 2) walking and encumbered. A two-dimensional (2-D) Fitts' Law task was used to examine tapping and dragging while one-dimensional (1-D) Fitts' Law targeting tasks were designed to measure spreading \& pinching and the two rotating gestures. We begin by explaining the use of Fitts' Law as a method to measure the performance of each individual gesture. We then describe the design of our tasks and the approach used to evaluate the different gestures.

\section{Using Fitts' Law to Evaluate Touchscreen Gestures}

Originally, Fitts' Law [7] characterised the performance of a one-dimensional pointing task. Participants move rapidly between two targets where target width and distance are controlled. Fitts showed that movement time had a strong correlation with the target distance to target width ratio. Fitts' Law has the form:

$$
M T=a+b * I D, I D=\log _{2}(A / W+1)
$$

Where $\boldsymbol{M T}$ is the movement time, $\boldsymbol{a}$ and $\boldsymbol{b}$ are constants determined by linear regression, $\boldsymbol{I D}$ is the Index of Difficulty and, in the Shannon formulation [18] used here, $\boldsymbol{A}$ is the target distance and $\boldsymbol{W}$ is the target width. Since high error rates are predicted when walking and encumbered, and the formula stated above assumes an error rate of $4 \%$, the effective target width $\left(\boldsymbol{W}_{\boldsymbol{e}}\right)$ is used instead. For the 1-D targeting task, $\boldsymbol{W}_{\boldsymbol{e}}$ is calculated as: SD $* 4.133$ [18], where SD is the standard deviation of the endpoint errors (i.e. the univariate endpoint deviation). More recently, Wobbrock et al. [30] showed that this method of calculating $\boldsymbol{W}_{\boldsymbol{e}}$ for 2-D targeting tasks is less appropriate and the spread of hits around their centre of mass should be used (i.e. the bivariate endpoint deviation). We therefore used Wobbrock et al.'s method (see [30] for details) to calculate $\boldsymbol{W}_{\boldsymbol{e}}$ for the 2-D tasks in our study. After $\boldsymbol{W}_{\boldsymbol{e}}$ was adjusted, the effective Index of Difficulty $\left(I D_{e}\right)$ was calculated using $\log _{2}\left(A / W_{e}+1\right)$.

Throughput (TP) [16] of each type of gesture was calculated as: $\boldsymbol{I D} \boldsymbol{D}_{\text {mean }} \boldsymbol{M} \boldsymbol{M}_{\text {mean }}$, where $\boldsymbol{I D}$ mean is the Index of Difficulty (calculated from the mean $\boldsymbol{I D}$ using $\boldsymbol{W}_{\boldsymbol{e}}$ ) and $\boldsymbol{M} \boldsymbol{T}_{\text {mean }}$ is the mean Movement Time. Throughput (in bits/sec) is normally used to compare the performance of different pointing devices but we calculated it to show the bandwidth of the communication channel of each type of gesture.

The use of Fitts' Law in HCI is not limited to pointing and has been used for other touch-based gesture types. Mackenzie et al. [17] used it to evaluate different input devices for tapping and dragging tasks. Tran et al. [29] modelled 
the performance of pinching and spreading gestures on mobile devices using a Fitts' Law style task. Zhao et al. [31] combined Fitts' Law with the Mahalanobis distance metric to evaluate translocation, rotation, and scaling movements on a multi-touch tabletop. Since the gesture types were combined, their method makes it difficult to discretely measure the performance of rotating gestures. The approach we used allowed us to examine two-finger rotating actions separately from the other gesture forms.

\section{Experimental Tasks}

We designed a set of Fitts' Law style targeting tasks to test each gesture type. Each trial was completed as quickly and accurately possible. No feedback was given to indicate a correct target selection in any of our tasks to avoid any influences on input behaviour [4]. The range of IDs was kept similar (as much as possible) across all tasks to define the target widths and distances for each gesture type. All tasks ran on a Samsung Galaxy S3 phone, which has a touchscreen resolution of $720 \mathrm{x} 1280 \mathrm{px}(\sim 12.05 \mathrm{px} / \mathrm{mm})$.

A two-handed input posture was used where the device was held in the non-dominant hand in portrait orientation and the dominant hand was used to perform the gestures. While users may switch to one-handed interaction when encumbered, during pilot tests we found that it was very difficult to perform long vertical dragging actions along the touchscreen and two-finger spreading, pinching and rotating gestures successfully in one continuous motion with one hand only. In addition, larger mobile phones, such as Apple's iPhone 6 Plus, are becoming more popular so interactions are likely to require both hands. Therefore, we picked a common two-handed input posture [11] to test the effects of encumbrance on each gesture. $\mathrm{Ng}$ et al. [22] previously compared one- and two- handed targeting when encumbered and results showed that tapping performance between the two poses were comparable.

\section{Tapping and Dragging}

To measure tapping performance, two targets (denoted as start and destination) were presented on screen. The start target was represented by a crosshair and had a diameter of $2.5 \mathrm{~mm}$. The size of the start target stayed constant for each trial and was chosen after pilot tests. The destination target was shown as a green circle and varied in width depending on the experimental trial. We chose this implementation to avoid confusion and decrease the chance of users selecting the wrong initial target. The index finger tapped the crosshair and then the destination target. As a result, two taps were required to complete each trial. Movement time for each tapping trial was the duration between the first touch down and second touch up events. For dragging, the same task was used but instead of two taps, the index finger selected the crosshair and dragged towards the destination target. Movement time for each dragging trial was the duration between the touch down and touch up events. Figure 2 shows the task used for tapping and dragging.
There were 3 target widths $(5.0,7.5$ and $10.0 \mathrm{~mm}), 4$ target distances $(24,36,48$ and $96 \mathrm{~mm}), I D$ ranged from 1.8 to 4.3 bits and 8 directions (N-S, NE-SW, E-W, SE-NW, S-N, SW-NE, W-E and NW-SE). There were 90 unique trials instead of 96 because the E-W and W-E directions are not possible for the largest distance of $96 \mathrm{~mm}$ due to the width of the touchscreen. The distances were selected to cover a wide area of the screen. The target widths represented a range of differently sized icons or buttons that users would typically press on mobile phones.

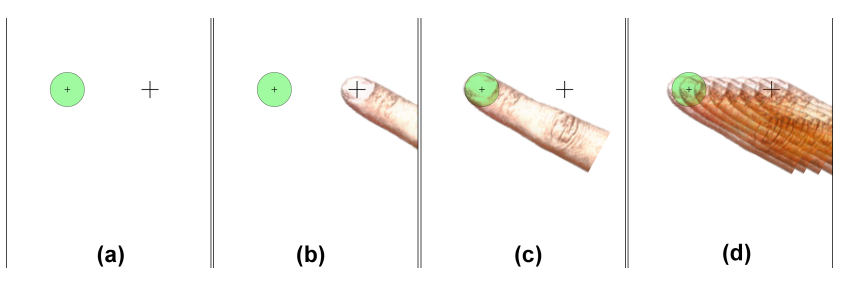

Figure 2. The task used for tapping and dragging (a). For tapping, the first tap selects the crosshair (b) and the second tap selects the destination target (c). For dragging, the initial crosshair is selected and the finger dragged towards the destination target $(d)$.

Spreading and Pinching

To examine two-finger spreading and pinching gestures, a similar method to Tran et al. [29] was developed. Circular targets were used instead of squares for better visual presentation (Findlater et al. [6] also used circles). For spreading, a circle (denoted as the control) was initially presented at the centre of the screen to show the current trial was ready (yellow). Once the index finger and the thumb (only these digits were used for input) of the dominant hand were placed on the touchscreen, the control circle turned green to show that the trial could begin. Participants were then instructed to move the control circle towards the target (grey ring) by expanding the distance between the digits. Like [29], a 1:1 mapping was used to transform the change in distance between the digits to the change in the size of the control circle. A trial ended once either digit lifted off the screen. There were no fixed starting points defined for each digit, but participants were asked to avoid placing their digits too close together at the start to prevent occlusion of the control circle.

The task for pinching operated in the same way as spreading, but the control circle was now initially bigger than the target. Like spreading, there were no fixed starting positions but the participants were instructed to touch the outer white area of the target at the start to ensure that there was enough space between the digits to perform the gesture. Figure 3 illustrates the tasks used for spreading and pinching. Each spreading and pinching gesture had to be completed in one single action and no correction was allowed if participants overshot. Movement time for both spreading and pinching was calculated from the touch down event of the second digit to the first touch up event of either digit. The same target widths and gesture distances as [29] were used, thus, there were 3 target widths (1.6, 3.2 and $4.8 \mathrm{~mm})$, 
3 gesture distances $(8,16$ and $24 \mathrm{~mm})$ and $I D$ ranged from 1.4 to 4.0 bits. Target distance $(A)$ in this case was from the edge of the control circle to the centre of the target ring.

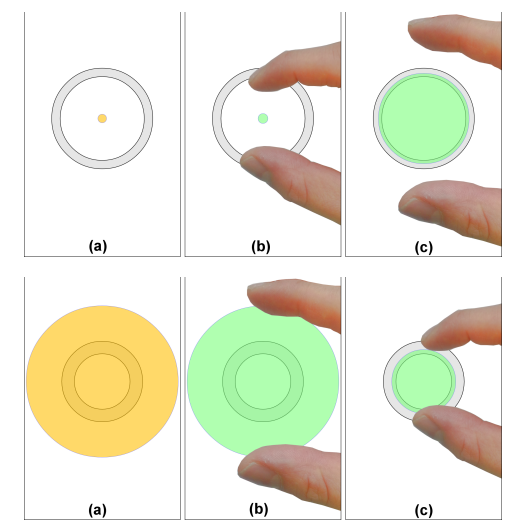

Figure 3. The tasks (a) used for spreading (top) and pinching (bottom). Once both digits were detected, the control circle turned green and the task began (b). A successful selection (c).

\section{Rotating}

Figure 4 explains our method of measuring two-finger rotating gestures. The touchscreen was split into two sections. The upper part was used for visual feedback, which consisted of an arc segment (limited to $110^{\circ}$ ) to show the amount of angle rotated and required to reach the green destination target. Note that the amount of angle shown in the arc directly corresponded to the on-screen movement, but we decided to shift visual feedback upwards slightly to avoid occluding the target location so that participants always knew where to rotate to and the amount of rotation required. This is similar to rotating a map, for example, where the user sees the area around and above their fingers as they rotate the view. The lower light blue area was used to perform the rotational gestures (Fig 4a).

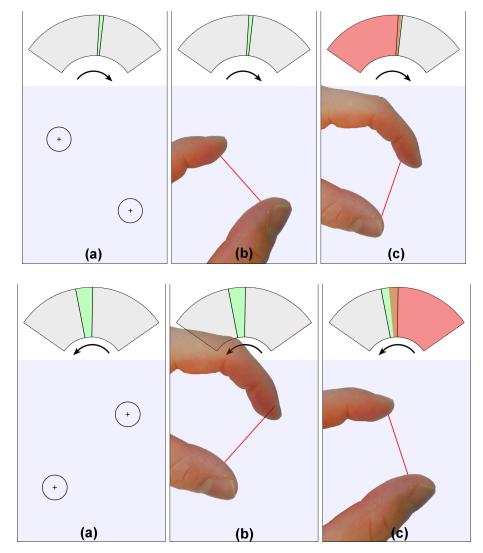

Figure 4. The task (a) used for rotating clockwise (top) and anticlockwise (bottom). Once both digits have been detected (b), the fingers rotated towards the green target (c).

There were two start points (both consisting of a circle $\&$ a crosshair): one for the index finger (upper left) and one the thumb (lower right) for rotating clockwise. The index finger and thumb were placed upper right and lower left posi- tions respectively for rotating anticlockwise. The distance between the start points was fixed at $41.5 \mathrm{~mm}$ for each trial since this was found to be a comfortable posture during pilot tests. Once both digits had been detected, a red line between the two touch positions appeared to show the task could begin (Fig 4b). The participants were asked to perform the action towards the green target area (shown in the feedback arc). Continuous feedback (in transparent red) was given in the arc segment as the fingers executed the rotational gesture $(\mathrm{Fig} 4 \mathrm{c})$. A 1:1 mapping was used to translate the amount of angle rotated to the progression of the feedback. For both rotational directions, the initial touch down co-ordinates of each digit had to be within their starting positions to avoid physical stresses on the user's fingers when performing the largest rotational distances. If this alignment did not occur, the gesture area turned red and both digits had to lift off the screen and accurately reselect the starting points again.

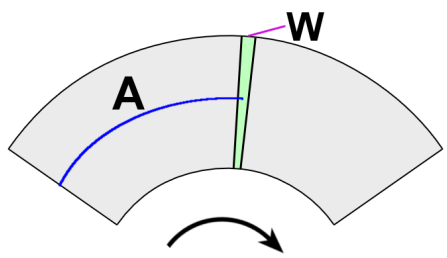

Figure 5. The definition of distance $(A)$ and target width $(W)$ for the rotational task (clockwise).

Several design features were carefully considered in our rotational task: 1) the main objective was to examine rotations independently and therefore we focused on the rotational angle. Participants could vary the gap between the digits (when rotating) without affecting the angle, as occurs in standard touchscreen rotations (and was done by Hoggan et al. [9]). 2) The maximum rotational angle was limited to $110^{\circ}$ from the starting angle (the difference in angle from the horizontal $\mathrm{x}$-axis) that was calculated between the index finger and the thumb. We found during pilot tests that this range was the physical limit before clutching was required. 3) All rotating gestures were performed in one single action, which is in accordance with a Fitts' Law style targeting task. 4) Both digits had to move in the rotational direction required to complete the gesture.

Each trial ended once either digit lifted off the screen. Movement time was calculated from the touch down event of the second digit to the first touch up event of either digit. Target width was defined as the green target area in the feedback arc while target distance was the amount of rotational angle required from the initial angle (calculated from the start touch down positions) to the centre of the target. Both target distance and target width were measured in degrees (see Figure 5). There were 3 target widths $\left(6^{\circ}, 12^{\circ}\right.$ and $\left.18^{\circ}\right), 3$ rotational distances $\left(30^{\circ}, 60^{\circ}\right.$ and $\left.90^{\circ}\right)$ and $I D$ ranged from $1.4-4.0$ bits. We chose these target widths and distances to cover a range of rotational precision that users might encounter with touchscreen mobile phones. 


\section{Encumbrance and Walking Scenario}

We selected a similar encumbrance scenario to $\mathrm{Ng}$ et al. [22] to replicate situations where both hands are physically hampered (see right image of Figure 1). Therefore, a typical carrier bag weighing $1.6 \mathrm{~kg}$ was held in each hand. The dimensions ( $\mathrm{w} x \mathrm{~h} x \mathrm{~d}$ ) of each bag were $330 \times 420 \times 90 \mathrm{~mm}$. While various encumbrance scenarios could have been evaluated, the main focus of our study was to examine the performance of the standard forms of touch-based gestures. So to keep the number of experimental conditions down, we decided to focus on one common situation where the user is carrying multiple objects in both hands.

To evaluate the effects of walking, we used a similar procedure to $\mathrm{Ng}$ et al. [22,23] where an oval route $(20 \mathrm{~m}$ in length) was set up in a quiet room. Each participant's preferred walking speed during interaction $(P W S \& I)$ was recorded at the beginning of the experiment and he/she had to maintain this speed during each condition by walking sideby-side with a human pacesetter (for more details, see [23]). As explained by $\mathrm{Ng}$ et al., when the two physical factors are not controlled, it is difficult to interpret the results since the effects of mobility are mixed up with encumbrance. Using the pacesetter approach meant that walking speed could not be traded with interaction (i.e. slowing down to input more precisely) and any effects observed are due to encumbrance since walking speed was controlled. Previous mobile studies (e.g. [8,12,24]) have also used a pacesetter to regulate walking speed for similar reasons.

\section{Experimental Design}

Twenty students (15 males, 5 females), aged between 21 and 38 years $($ mean $=26.15, \mathrm{SD}=4.09)$, recruited from the university took part in the study. Three of the male participants preferred using their left hand for input while all female participants were right-handed. All participants used a touchscreen mobile phone or device on a daily basis. The experiment took around 90 minutes to complete and each participant was paid for taking part. To reduce fatigue, sufficient resting periods were given between conditions and as required by the participants. A training phase for each gesture was given at the start of the experiment to familiarise the participants with the different tasks.

There were six gesture conditions: tapping, dragging, spreading, pinching, rotating clockwise and rotating anticlockwise. For tapping and dragging, each block of trials consisted of the 90 target width/distance combinations. For spreading and pinching, each block of trials had 45 target selections since each of the 9 target width/distance combinations was repeated five times. This was the same for rotating clockwise and rotating anticlockwise. The order of the trials within each block was randomised and an arbitrary delay between 500 - 1500ms was placed between each trial to reduce the chance of any rhythm forming between input and gait [5]. There were two blocks of trials per condition therefore each participant completed 1440 target selections.
Each gesture was completed while walking and either unencumbered or holding the bags, which resulted in a total of 12 conditions. The conditions were counterbalanced by type of encumbrance and the order of the gestures was further randomised. The Independent Variables were type of gesture, type of encumbrance and target width/distance combination. The Dependent Variables were target accuracy $(\%)$ and movement time (milliseconds). The hypotheses were:

H1: For each type of gesture, accuracy will be significantly lower when carrying the bags than unencumbered;

$H 2$ : For each type of gesture, movement time will take significantly longer when carrying the bags than unencumbered;

H3: Dragging will have significantly higher accuracy than tapping but significantly slower movement time (due to the findings of [4]);

H4: Pinching will be performed significantly better than spreading (based on the outcomes of $[10,14]$ );

H5: Rotating antiCW will be performed significantly better than rotating $C W$ (due to the results of [9]).

\section{RESULTS}

The mean target accuracy and selection time for each condition are shown in Figure 6 and Figure 7 respectively.

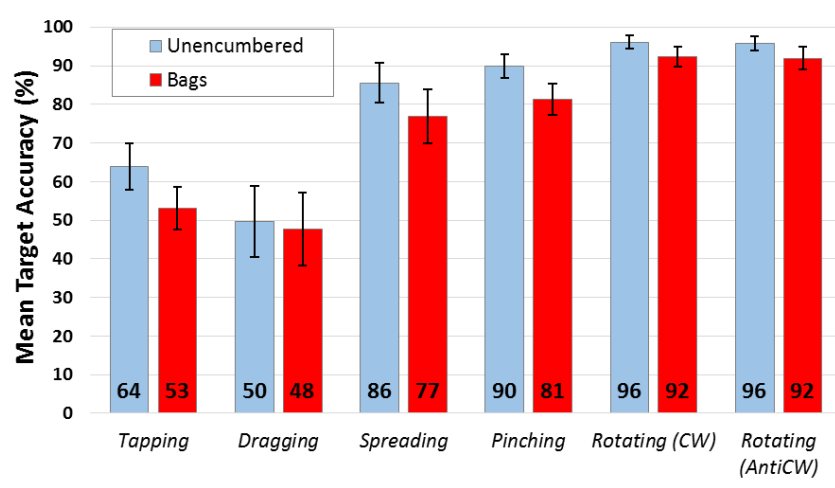

Figure 6. The overall mean accuracy (\%) for each condition. Error bars denote $95 \%$ CI.

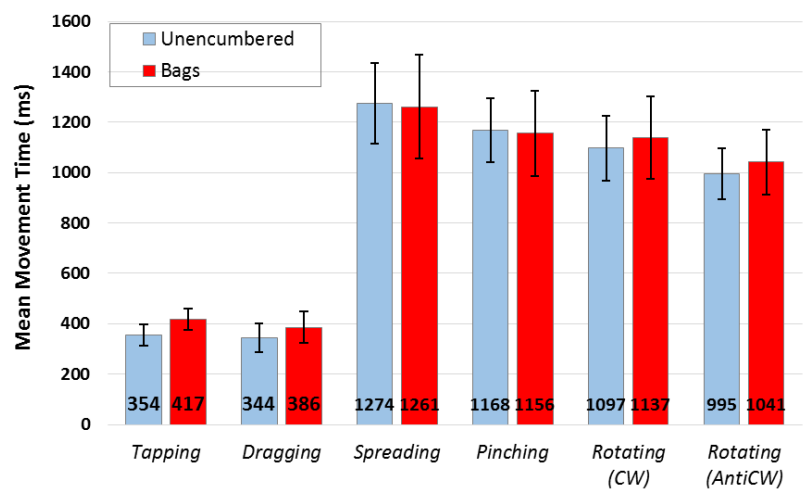

Figure 7. The overall mean movement time $(\mathrm{ms})$ for each condition. Error bars denote $95 \%$ CI. 
A total of 28,800 trials were recorded for the entire experiment. Potential outliers were removed (by following the method described by [16]) if 1) the measured movement was less than half of the distance to the target $(A)$ (only applicable for tapping and dragging) or 2) the endpoint error was greater than two target widths $(2 W)$ from the centre of the current target (used for all types of gestures). As a result, 494 trials were deemed as outliers (1.7\%) and were removed from the final data analysis. Three-factor (type of gesture, type of encumbrance and target width/distance combination) repeated-measures ANOVAs were conducted for target accuracy and movement time to compare tapping vs. dragging, spreading vs. pinching and rotating clockwise vs. anticlockwise. Greenhouse-Geisser adjustments were used to correct the degrees of freedom each time that Mauchly's test for sphericity was significant.

\section{Tapping and Dragging}

The ANOVA for accuracy showed a significant main effect for type of gesture, $F(1,19)=6.03, p<0.05$. Accuracy was significantly higher for tapping than dragging by $9.8 \%$. There was a significant main effect for type of encumbrance, $F(1,19)=17.69, p<0.01$. The number of correct target selections was significantly higher when unencumbered than holding the bags, a difference of $6.4 \%$. A significant main effect was observed for target combination, $F(11,209)=105.74, p<0.01$. Post hoc pairwise comparisons with Bonferroni corrections showed that for each target distance, increasing target width significantly increased target accuracy. The interaction between type of gesture and type of encumbrance was significant, $F(1,19)=14.37$, $p<0.01$. Encumbrance caused a greater decrease in accuracy when tapping than dragging. Target accuracy was low for dragging whether participants were encumbered or not. The interaction between type of gesture and target combination was significant, $F(5.2,98.1)=11.94, p<0.01$. Accuracy for all three target widths at the largest distance $96 \mathrm{~mm}$ was significant higher when dragging than tapping. The accuracy for all other target combinations was higher for tapping than dragging. The interaction between type of encumbrance and target combination was not significant, $p$ $>0.05$. The interaction between all three factors for accuracy was also not significant, $p>0.05$.

The ANOVA for movement time showed no significant main effect for type of gesture, $p>0.05$. A significant main effect was found for type of encumbrance, $F(1,19)=$ 12.95, $p<0.01$. Movement time took significantly longer when holding the bags than when unencumbered (a mean difference of $52.8 \mathrm{~ms}$ ). A significant main effect was observed for target combination, $F(1.6,30.8)=219.84, p<$ 0.01. Post hoc pairwise comparisons with Bonferroni corrections showed that increasing target width did not have a significant effect on movement time at each target distance. However, increasing target distance significantly increased movement time for each target width. The interaction between type of gesture and type of encumbrance was not significant, $p>0.05$. The interaction between type of ges- ture and target combination was significant, $F(2.7,51.7)=$ $7.19, p<0.01$. Movement time for all target widths at the greatest distance of $96 \mathrm{~mm}$ took significantly longer for dragging than tapping. However, movement time for the other nine target combinations was significantly faster for dragging than tapping. The interaction between type of encumbrance and target combination was significant, $F(3.0$, $57.6)=5.59, p<0.01$. Carrying the bags caused significantly longer movement time for all target combinations than unencumbered. The interaction between all three factors for movement time was not significant, $p>0.05$.

\section{Spreading and Pinching}

The ANOVA for accuracy showed no significant main effect for type of gesture, $p>0.05$. There was a significant main effect for type of encumbrance, $F(1,19)=50.59, p<$ 0.01 . Target accuracy was significantly higher when unencumbered than holding the bags, a mean difference of $8.7 \%$. A significant main effect for target combination was also observed, $F(3.9,74.5)=62.17, p<0.01$. Post hoc pairwise comparisons with Bonferroni corrections showed that accuracy significantly increased as target width also increased at each gesture distance. No significant effect was observed for the interaction between type of gesture and type of encumbrance, $p>0.05$. The interaction between type of gesture and target combination was significant, $F(3.3,63.4)=$ $4.2, p<0.01$. Accuracy was lower for $1.6 \mathrm{~mm}$ and $3.2 \mathrm{~mm}$ target widths at the largest gesture distance of $24 \mathrm{~mm}$ when pinching than spreading. The accuracy for all other target combinations was significantly higher for pinching than spreading. The interaction between type of encumbrance and target combination was not significant, $p>0.05$. No significant effect was found between all three factors for accuracy, $p>0.05$.

The ANOVA for movement time showed a significant main effect for type of gesture, $F(1,19)=7.57, p<0.05$. Movement time was significantly faster by $106 \mathrm{~ms}$ when pinching than spreading. No significant main effect was observed for type of encumbrance, $p>0.05$. There was a significant main effect for target combination, $F(1.6,30.3)$ $=56.76, p<0.01$. Post hoc pairwise comparisons with Bonferroni corrections showed that movement time was significantly faster as target width increased at each gesture distance. Also, movement time took significantly longer as gesture distance increased for each target width. The interaction between type of gesture and type of encumbrance was not significant, $p>0.05$. A significant effect was found for the interaction between type of gesture and target combination, $F(3.5,66.0)=4.36, p<0.01$. The movement time for target combination $3.2 / 8.0 \mathrm{~mm}$ was slower for pinching than spreading. All other target combinations were selected significantly faster when pinching than spreading. The interaction between type of encumbrance and target combination was not significant, $p>0.05$. The interaction between all three factors for movement time was also not significant, $p>0.05$. 


\section{Rotating}

The ANOVA for accuracy showed no significant main effect for type of gesture, $p>0.05$. There was a significant main effect for type of encumbrance, $F(1,19)=34.74, p<$ 0.01 . Accuracy was significantly higher when unencumbered than holding the bags (a mean difference of 3.7\%). A significant main effect was also found for target combination, $F(2.2,42.3)=21.84, p<0.01$. Post hoc pairwise comparisons with Bonferroni corrections showed that accuracy significantly increased as target width also increased at each rotational distance. However, the mean difference between target width $12^{\circ}$ and $18^{\circ}$ was small. The interaction between type of gesture and type of encumbrance was not significant, $p>0.05$. No significant interaction was observed between type of gesture and target combination, $p$ $>0.05$. The interaction between type of encumbrance and target combination was significant, $F(4.9,83.4)=7.48, p<$ 0.01 . Accuracy for target combination $18^{\circ} / 90^{\circ}$ was higher when holding the bags than when unencumbered. The interaction between all three factors was not significant, $p>$ 0.05 .

The ANOVA for movement time showed a significant main effect for type of gesture, $F(1,19)=9.54, p<0.01$. Movement time for rotating anticlockwise was significantly faster than rotating clockwise, a small mean difference of $99 \mathrm{~ms}$. There was no significant main effect for type of encumbrance, $p>0.05$. A significant main effect was observed for target combination, $F(1.6,29.5)=86.28, p<$ 0.01. Post hoc pairwise comparisons with Bonferroni corrections indicated that movement time was significantly faster as target width increased at each rotational distance. Movement time took significantly longer as rotational distance increased for each target width. The interaction between type of gesture and type of encumbrance was not significant, $p>0.05$. A significant interaction was observed between type of gesture and target combination, $F(1.8,33.3)=4.55, p<0.05$. The decrease in movement time between rotating clockwise and anticlockwise for all three target widths at the largest rotational distance was significantly greater than for the other six target combinations. The interaction between type of encumbrance and target combination was significant, $F(4.1,78.4)=3.08, p<$ 0.05 . The movement time of target $6^{\circ} / 30^{\circ}$ was significantly quicker when holding the bags than when unencumbered. All other target combinations took significantly longer to select when encumbered. The interaction between all three factors for movement time was not significant, $p>0.05$.

\section{Fitts' Law Analysis}

Table 1 shows the calculated values for $\boldsymbol{a}$ and $\boldsymbol{b}$, correlation coefficient $(\boldsymbol{r})$ and coefficient of determination $\left(\boldsymbol{R}^{2}\right)$ for each condition. A strong correlation $(>0.9)[18,29])$ indicates the possible use of our tasks to model the performance of each gesture type. The Throughput $(\boldsymbol{T P})$ for each condition is also presented. Figure 8 shows a plot of $\boldsymbol{M T}$ against $\boldsymbol{I D}_{\boldsymbol{e}}$ for each gesture type. The correlation values in Table 1 indicate a strong linear relationship for tapping, dragging and rotating anticlockwise in each encumbrance scenario. A weaker relationship is shown for spreading, pinching and rotating clockwise which suggests more varied performance when users were walking and carrying the bags. In terms of $\boldsymbol{T P}$, for each gesture type, a higher rate of information transfer was found when unencumbered than when both hands were encumbered.

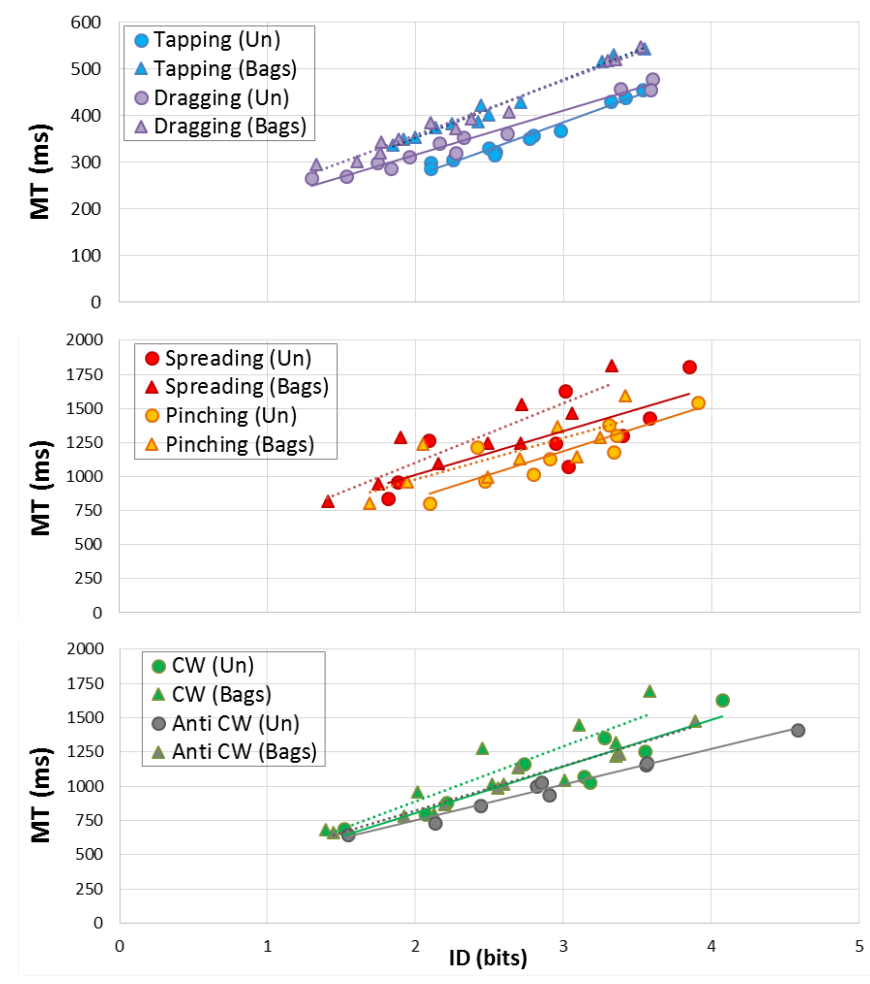

Figure 8. Plot of $M T$ vs. $I D_{e}$ for tapping \& dragging (top - note the different $y$-axis units), spreading \& pinching (middle), and rotating clockwise \& anticlockwise (bottom).

\begin{tabular}{|l|c|c|c|c|c|}
\hline \multicolumn{1}{|c|}{ Condition } & a & b & r & $\mathbf{R}^{2}$ & TP \\
\hline Tapping (Un) & 40.5 & 114.9 & 0.98 & 0.97 & 5.58 \\
\hline Tapping (Bags) & 102.5 & 124.8 & 0.99 & 0.98 & 4.27 \\
\hline Dragging (Un) & 128.6 & 93.9 & 0.99 & 0.97 & 4.82 \\
\hline Dragging (Bags) & 122.9 & 117.4 & 0.99 & 0.98 & 4.16 \\
\hline Spreading (Un) & 372.9 & 320.5 & 0.78 & 0.60 & 2.23 \\
\hline Spreading (Bags) & 235.1 & 434.1 & 0.90 & 0.82 & 1.87 \\
\hline Pinching (Un) & 153.3 & 344.6 & 0.88 & 0.77 & 2.53 \\
\hline Pinching (Bags) & 371.0 & 304.7 & 0.79 & 0.63 & 2.24 \\
\hline Clockwise (Un) & 122.7 & 340.7 & 0.93 & 0.86 & 2.60 \\
\hline Clockwise (Bags) & 86.5 & 401.3 & 0.88 & 0.77 & 2.32 \\
\hline Anticlockwise (Un) & 225.0 & 262.4 & 0.99 & 0.98 & 2.91 \\
\hline Anticlockwise (Bags) & 174.5 & 324.4 & 0.99 & 0.97 & 2.54 \\
\hline
\end{tabular}

Table 1. The values of $a, b, r, R^{2}$ and $T P$ for each gesture/encumbrance combination. 


\section{DISCUSSION}

The results for target accuracy showed that the number of correct selections significantly decreased when encumbered for each gesture type and therefore hypothesis $H 1$ is supported. For tapping, the mean accuracy while walking and unencumbered was $65 \%$ and dropped to $53 \%$ when the bags were held. The previous encumbered and walking study of $\mathrm{Ng}$ et al. [22] reported similar results when users in their experiment performed a non-Fitts' Law targeting task in the same input posture. Dragging while carrying the bags caused the lowest mean accuracy of $48 \%$ across all the conditions. However, unencumbered dragging was only $2 \%$ better, which suggests walking had a greater effect on accuracy than encumbrance in this case. Encumbrance caused a drop in overall accuracy of $9 \%$ for both spreading and pinching. However, both types of gestures performed surprisingly well as we expected the participants to make more incorrect selections in the tested situations. Likewise, the rotating clockwise and anticlockwise gestures were executed very well (all accuracies $>90 \%$ ). Both rotational input types had identical mean accuracies when unencumbered and carrying the bags. In addition, encumbrance only decreased accuracy by $4 \%$, which gives some indications that rotating gestures are more resilient to the effects of encumbrance than the other types of gestures while on the move.

In terms of movement time, target selections took significantly longer when encumbered for both tapping and dragging. However, holding the bags did not significantly increase movement time when compared to unencumbered for spreading, pinching and rotating in both directions. Therefore, hypothesis $H 2$ can only be partially supported. The overall mean movement times for tapping and dragging increased by $18 \%$ and $12 \%$ respectively when the bags were held. The mean movement times were very similar between unencumbered and carrying the bags for both spreading and pinching as differences were $\sim 1 \%$. The difference in mean movement time between unencumbered and holding the bags for both rotating clockwise and anticlockwise was less than 5\%. The one-finger gestures took on average less than $500 \mathrm{~ms}$ to execute while the two-finger actions required more than $1000 \mathrm{~ms}$. Perhaps participants in our study traded accuracy for movement time when tapping and dragging or the nature of spreading, pinching and rotating movements simply require more time to perform.

Hypothesis $H 3$ is rejected since accuracy for tapping was significantly higher than dragging. Furthermore, there was no significant difference in movement time between tapping and dragging. Cockburn et al. [4] reported that touch dragging was slower but caused fewer errors than tapping on a tablet PC when users were stationary and unencumbered. However, the advantage of an offset cursor used in their study meant that users had the advantage of continual feedback. This is not normally available on touchscreen mobile phones and from our observations, participants found it difficult to know if a target was successfully selected due to visual occlusion by the input finger when drag- ging. All target widths at the largest distance of $96 \mathrm{~mm}$ were selected more accurately when dragging than tapping which suggests that dragging might be better than tapping for tasks that require high accuracy over longer distances.

The comparison between spreading and pinching showed no significant effect for target accuracy. However, movement time for pinching was significantly faster than spreading. Thus, hypothesis $H 4$ is partially supported. Previous studies have reported conflicting results between spreading and pinching in static contexts. Our results showed that spreading and pinching gestures were evenly matched when users were walking and encumbered. The overall mean unencumbered movement times for spreading and pinching while walking were $1274 \mathrm{~ms}$ and $1168 \mathrm{~ms}$ respectively. For comparison, Tran et al. [29] reported mean execution times of $1020 \mathrm{~ms}$ and $1090 \mathrm{~ms}$ for spreading and pinching respectively on a mobile phone when users were seated and unencumbered. Because Fitts' Law targeting tasks were used to measure the performance of each gesture type, users performed spreading and pinching in one continuous movement for each trial. Therefore, one limitation of our spreading and pinching tasks is that they do not take clutching into account. Avery et al. [1] showed that users clutched frequently when performing pinching to zoom into an area of interest. As a result, they developed Pinch-tozoom-Plus (PZP), which reduced the amount of clutching and panning required during pinch-to-zoom actions. Future work should test to see if PZP is still effective in walking and encumbered situations.

The results for the rotating gestures showed no significant difference in terms of accuracy between rotating clockwise and anticlockwise. However, movement time was significantly reduced for rotating anticlockwise than clockwise therefore hypothesis $H 5$ can only be partially supported. Our results were similar to Hoggan et al. [9], who reported that clockwise rotations took longer than anticlockwise ones for specific starting positions on a tablet while seated. Overall, the rotating actions were executed the most successfully in terms of accuracy out of all the gesture types we studied. Furthermore, prior to conducting statistical tests, we examined each participant's performance for each individual gesture due to potential input differences between left- and right- handed users, especially for executing rotational actions. Observing the data suggested that there was no great disparity in performance across all conditions regardless of the gestures completed using the left or right hand. We also asked the participants if they found the rotating gestures easier to perform in a particular direction. A majority of the participants commented that there was no preferred rotational direction only that carrying the bags made input subjectively more physically challenging to perform.

\section{CONCLUSIONS}

To conclude, the user study presented in this paper extends previous research on the effects of encumbrance by examin- 
ing the performance of standard touch-based gestures that have become common on touchscreen devices. A set of Fitts' Law style targeting tasks was designed to measure one-finger tapping and dragging, and two-finger spreading, pinching and rotating while walking and carrying typical shopping bags. Despite different targeting tasks were used, the results showed that two-finger gestures were performed more accurately than the one-finger input methods but movement time took substantially longer. Perhaps designing two-finger input techniques to give greater accuracy at the expense of longer execution times is preferable in encumbered and walking contexts. An inaccurate target selection can be even more costly in time due to error recovery and increases user frustration. However, one-finger interactions are inevitable, therefore small targets should be avoided if possible, especially when dragging due to the visual occlusion of targets by the input finger that causes ambiguous selections. Target accuracy did not exceed $90 \%$ with the largest target width tested for both tapping and dragging, thus bigger targets are required to further increase accuracy. We hope our study will motivate designers to think about the input techniques they might choose for interactions in applications designed to be used in everyday mobile settings where users are likely to be carrying things. Maybe, for example, instead of choosing a simple tap, an alternative design might use a two-finger rotation which would result in greater accuracy for users on the move and carrying objects.

\section{REFERENCES}

1. Avery, J., Choi, M., Vogel, D., and Lank, E. Pinch-tozoom-plus. Proceedings of UIST 2014, 595-604.

2. Barnard, L., Yi, J.S., Jacko, J.A., and Sears, A. An empirical comparison of use-in-motion evaluation scenarios for mobile computing devices. International Journal of Human Computer Studies 62, 4 (2005), 487520 .

3. Bergstrom-Lehtovirta, J., Oulasvirta, A., and Brewster, S. The effects of walking speed on target acquisition on a touchscreen interface. Proceedings of MobileHCI 2011, 143-146.

4. Cockburn, A., Ahlström, D., and Gutwin, C. Understanding performance in touch selections: Tap, drag and radial pointing drag with finger, stylus and mouse. International Journal of Human-Computer Studies 70, 3 (2012), 218-233.

5. Crossan, A., Murray-Smith, R., Brewster, S., Kelly, J., and Musizza, B. Gait Phase Effects in Mobile Interaction. Extended Abstracts on Human Factors in Computing Systems - CHI EA 2005, 1312-1315.

6. Findlater, L., Froehlich, J.E., Fattal, K., Wobbrock, J.O., and Dastyar, T. Age-related differences in performance with touchscreens compared to traditional mouse input. Proceedings of CHI 2013, 343-346.
7. Fitts, P.M. The information capacity of the human motor system in controlling the amplitude of movement. 1954. Journal of experimental psychology. General 121, 6 (1992), 262-269.

8. Goel, M., Findlater, L., and Wobbrock, J. WalkType: Using Accelerometer Data to Accommodate Situational Impairments in Mobile Touch Screen Text Entry. Proceedings of CHI 2012, 2687-2696.

9. Hoggan, E., Williamson, J., Oulasvirta, A., Nacenta, M., Kristensson, P.O., and Lehtiö, A. Multi-touch rotation gestures: performance and ergonomics. Proceedings of CHI 2013, 3047-3050.

10. Hoggan, E., Williamson, J., Oulasvirta, A., Nacenta, M., Kristensson, P.O., and Lehtiö, A. Multi-touch pinch gestures: performance and ergonomics. Proceedings of ITS 2013, 219 - 222.

11. Hoober, S. How Do Users Really Hold Mobile Devices? www.uxmatters.com

12. Kane, S.K., Wobbrock, J.O., and Smith, I.E. Getting off the treadmill: evaluating walking user interfaces for mobile devices in public spaces. Proceedings of MobileHCI 2008, 109-118.

13. Karlson, A., Bederson, B., and Contreras-Vidal, J. Understanding single-handed mobile device interaction. Technical Report 2006.

14. Kobayashi, M., Hiyama, A., Miura, T., Asakawa, C., Hirose, M., and Ifukube, T. Elderly user evaluation of mobile touchscreen interactions. Proceeding of INTERACT 2011, 83-99.

15. Lin, M., Goldman, R., Price, K.J., Sears, A., and Jacko, J. How do people tap when walking? An empirical investigation of nomadic data entry. International Journal of Human-Computer Studies 65, 9 (2007), 759-769.

16. MacKenzie, I.S. and Isokoski, P. Fitts' throughput and the speed-accuracy tradeoff. Proceedings of CHI 2008, $1633-1636$.

17. MacKenzie, I.S., Kauppinen, T., and Silfverberg, M. Accuracy measures for evaluating computer pointing devices. Proceedings of CHI 2001, 9-16.

18. MacKenzie, I.S. Fitts' Law as a Research and Design Tool in Human-Computer Interaction. HumanComputer Interaction 7, 1 (1992), 91-139.

19. Mainwaring, S.D., Anderson, K., and Chang, M.F. Living for the global city: mobile kits, urban interfaces, and ubicomp. Proceeding of UbiComp 2005, 269-286.

20. Mizobuchi, S., Chignell, M., and Newton, D. Mobile text entry: relationship between walking speed and text input task difficulty. Proceedings of MobileHCI 2005, 122-128. 
21. Ng, A., Brewster, S., and Williamson, J. The Impact of Encumbrance on Mobile Interactions. Proceeding of INTERACT 2013, 92-109.

22. Ng, A., Brewster, S.A., and Williamson, J.H. Investigating the effects of encumbrance on one- and two- handed interactions with mobile devices. Proceedings of CHI 2014, 1981-1990.

23. Ng, A., Williamson, J.H., and Brewster, S.A. Comparing evaluation methods for encumbrance and walking on interaction with touchscreen mobile devices. Proceedings of MobileHCI 2014, 23-32.

24. Nicolau, H. and Jorge, J. Touch typing using thumbs: understanding the effect of mobility and hand posture. Proceedings of CHI 2012, 2683 - 2686.

25. Oulasvirta, A. and Bergstrom-Lehtovirta, J. Ease of juggling: studying the effects of manual multitasking. Proceedings of CHI 2011, 3103-3112.

26. Schedlbauer, M. and Heines, J. Selecting While Walking: An Investigation of Aiming Performance in a Mobile Work Context Selecting While Walking: An Investigation of Aiming Performance in a Mobile Work Context. Proceedings of AMCIS 2007.

27. Schildbach, B. and Rukzio, E. Investigating Selection and Reading Performance on a Mobile Phone while Walking. Proceedings of MobileHCI 2010, 93-102.

28. Sears, A., Lin, M., Jacko, J., and Yang, X. When computers fade: Pervasive computing and situationally induced impairments and disabilities. Proceedings of HCI Int'l 2003, 1298 - 1302.

29. Tran, J.J., Trewin, S., Swart, C., John, B.E., and Thomas, J.C. Exploring pinch and spread gestures on mobile devices. Proceedings of MobileHCI 2013, 151160.

30. Wobbrock, J.O., Shinohara, K., and Jansen, A. The effects of task dimensionality, endpoint deviation, throughput calculation, and experiment design on pointing measures and models. Proceedings of $\mathrm{CHI}$ $2011,1639-1648$.

31. Zhao, J., Soukoreff, R.W., and Balakrishnan, R.A Model of Multi-touch Manipulation. Proceedings of GRAND 2011. 